Voix et Images

voixetimages

\title{
Le Cid et Hamlet : Corneille et Shakespeare lus par Ducharme et Gurik
}

\section{Renald Bérubé}

Volume 1, numéro 1, septembre 1975

Hubert Aquin

URI : https://id.erudit.org/iderudit/013984ar

DOI : https://doi.org/10.7202/013984ar

Aller au sommaire du numéro

Éditeur(s)

Les Presses de l'Université du Québec

ISSN

0318-9201 (imprimé)

1705-933X (numérique)

Découvrir la revue

Citer cet article

Bérubé, R. (1975). Le Cid et Hamlet : Corneille et Shakespeare lus par Ducharme et Gurik. Voix et Images, 1(1), 35-56. https://doi.org/10.7202/013984ar d'utilisation que vous pouvez consulter en ligne.

https://apropos.erudit.org/fr/usagers/politique-dutilisation/ 


\title{
Le Cid et Hamlet: Corneille et Shakespeare lus par Ducharme et Gurik
}

\begin{abstract}
Hamlet est comme une éponge. A la seule condition de ne pas le styliser ni le jouer comme une antiquité, il absorbe immédiatement tout notre temps ${ }^{1}$.
\end{abstract}

17 janvier 1968: première de Hamlet, prince du Québec de Robert Gurik; été 1968, création du Cid maghané de Réjean Ducharme à l'occasion du Festival de Sainte-Agathe. Bien sûr, le phénomène en soi n'est pas nouveau: les grandes œuvres classiques nous habitent tous plus ou moins, elles constituent tout à la fois des points d'appui et des points de repère. Les auteurs eux-mêmes n'échappent pas à la règle, en particulier les auteurs dramatiques: on n'en finirait pas de compter, au xxe siècle seulement, les reprises, par les auteurs les plus divers et selon les formes les plus diverses, de différentes œuvres classiques. Giraudoux, Sartre, Cocteau, Anouilh et O'Neill ont tous, dans une pièce ou dans une autre, "refait» une œuvre classique, présenté leur version d'un sujet dramatique connu et utilisé depuis longtemps.

La littérature québécoise $\mathrm{du} x \mathrm{x} \mathrm{x}^{\mathrm{e}}$ siècle a bien connu le phénomène de l'imitation: Louis Fréchette, par exemple, se voulait une sorte de copie conforme de son maître, Victor Hugo. Et la Légende des siècles devenait la Légende d'un peuple: Fréchette cherchait à s'identifier le plus totalement possible à Hugo; entre l'élève et le maître, aucune distance critique ne voulait ou ne pouvait trouver place, l'imitation tendait à devenir mimétisme 2 . Les choses ont certes changé depuis, les créateurs québécois ayant graduellement acquis des certitudes et une autonomie qui ne pouvaient être les leurs au milieu du siècle dernier. Quand Robert Gurik et Réjean Ducharme nous proposent un Hamlet et un Cid en 1968, ils ne sont pas à la remorque des cuvres dont ils s'inspirent. Ils nous en proposent une lecture personnelle qui n'est pas sans soulever de multiples points d'interrogation.

II convient d'abord de noter ceci: les œuvres qui servent de point de départ à Gurik et à Ducharme, celles de Shakespeare et de Corneille, furent elles-mêmes, en leurs temps, des adaptations personnelles de pièces ou de sujets connus ou à la mode ${ }^{3}$. Comme quoi ces œuvres reposent sur des scénarios qui, depuis longtemps déjà, appartiennent à tout le monde. La question ne se pose pas moins: pourquoi, en 1968, année particulièrement faste pour la jeune dramaturgie québécoise ${ }^{4}$, Gurik et 
Ducharme choisissent-ils, pour nous parler de nous, d'adapter une pièce anglaise de l'époque élizabéthaine et une pièce française du début du siècle classique? En d'autres termes, quelle lecture font-ils de ces pièces qui leur permette de donner à celles-ci une résonance à laquelle nous soyons particulièrement sensibles, qui leur permette de rendre compte de ce que nous sommes et de notre situation?

Après avoir dégagé sommairement les significations essentielles du Cid de Corneille à partir de l'analyse qu'en fait Serge Doubrovsky5, nous analyserons la pièce de Ducharme pour voir de quelle manière et dans quelle mesure elle copie, adapte, transforme, «parodie» l'cuvre de Corneille. Partant des constatations qui se dégageront de l'analyse, nous verrons quelle peut être la signification de la pièce de Ducharme. Le procédé sera le même pour Hamlet; nous retiendrons surtout, pour la pièce de Shakespeare, les analyses de Jean Paris ${ }^{6}$.

\section{LE CID}

Le fonne c'est platte

(la chair est triste et j'ai vu tous les films de Jerry Lewis) ${ }^{7}$.

\section{A. LE PROJET CORNÉLIEN DANS LE CID, SELON DOUBROVSKY}

Après avoir fait remarquer que les deux premières scènes du Cid, continuant en cela les cuvres antérieures de Corneille, sont des scènes de comédie ( $C D H$, p. 87), Doubrovsky en arrive rapidement à parler du monde médiéval: “... on n'a plus à revenir aujourd'hui sur le fait que le Cid exprime les préoccupations profondes d'une aristocratie à la recherche de ses origines féodales» $(C D H$, p. 89$)$; en cela, le théâtre de Corneille, s'il est un théâtre d'histoire, est plus justement un "théâtre de réaction contre l'histoire, théâtre réactionnaire » $(\mathrm{CDH}, \mathrm{p} .96)$.

Le monde féodal repose sur la loi du plus fort: dans sa dispute avec Don Diègue, le Comte ne met jamais en doute la grandeur passée de ce dernier. "Ce qui compte, c'est que sans rival aujourd'hui, le Comte se voit, contre toute attente, préférer un rival d'hier ; [...] leur conflit éclate lorsqu'il devient nécessaire d'instituer un ordre de préséance temporel " (CDH, p. 90). Puisqu'il est le plus fort aujourd'hui, le Comte aurait dû être choisi par le Roi.

Dans cette optique, la vieillesse devient donc le danger le plus grave, la menace à écarter: réduisant la force physique, la vieillesse risque même de ternir l'éclat des gestes passés et de faire du Maître un Esclave ( $C D H$, p. 92 et suiv.). Pour échapper au déshonneur (le soufflet qu'il a reçu du Comte) et vaincre la vieillesse, Don Diègue trouvera une solution: son fils est sa continuation, son fils les vengera. Rien ne dit mieux cette 
conception du temps et de la personne que le vers par lequel on annonce au Roi la mort du Comte:

Don Diègue, par son fils, a vengé son offense (le Cid, v. 633)

Rodrigue, essentiellement, y est décrit comme un fils, comme un instrument jeune qui agit à la place d'un père devenu trop âgé pour se défendre.

Pourtant, Rodrigue est loin d'être en accord avec toutes les idées de son père: la longueur et la structure des stances témoignent de ses hésitations et du pénible cheminement de sa pensée. À la scène 6 de l'acte III, il répondra:

L'infamie est pareille, et suit également

Le guerrier sans courage et le perfide amant

à son père qui vient d'affirmer:

Nous n'avons qu'un honneur, il est tant de maîtresses!

En fait, Rodrigue demande à son père de reconnaître le caractère unique de tout amour et donc son invidualité personnelle: Rodrigue refuse de n'être qu'un fils aux ordres du père. S'il a obéi au *Meurs ou tue " (le Cid, v. 275) de ce dernier, s'il a vaincu la peur de la mort afin de ne pas devenir l'Esclave de Don Gomès $(C D H, p$. 95), Rodrigue sait que son aventure n'est pas terminée: "Chez le Maître, la tendresse autant que la vaillance trouve son épreuve authentique dans l'affrontement mortel» (CDH, p. 105). C'est le sens des célèbres rencontres Rodrigue-Chimène: celle-ci, ne pouvant renoncer au désir de la possession physique de son amant (CDH, p. 106 et suiv.), ne pouvant affronter ni la mort de ce dernier ni la sienne, devient en quelque sorte, malgré ses paroles (qui ne se traduisent jamais par des actes), l'Esclave de Rodrigue. Mais si peu: Rodrigue ayant, par ses exploits, succédé à Don Gomès, Chimène ne trahit ni sa classe ni même sa race en l'épousant. Bien au contraire, elle assure la pérennité de celles-ci.

Vainqueur du Comte et de Chimène, Rodrigue accèdera bientôt, par sa victoire sur les Maures, à l'Histoire; plus, à la légende. Désormais appelé le Cid, il cessera d'être un «invaincu " pour devenir un «invincible». Le temps désormais ne saurait l'atteindre ni le détruire.

\section{B. LE CID MAGHANÉ: ÉLÉMENTS D'ANALYSE}

«Parodie en 14 rideaux écrite pour être jouée en costumes d'époque dans des meubles $1967^{\circ}$ ", le Cid maghané, dans son déroulement général, suit de très près l'œuvre de Corneille: dans l'une et l'autre pièce, les différentes scènes sont ordonnées de la même façon, malgré le fait que les cinq actes de la pièce de Corneille deviennent quatorze rideaux dans la parodie de Ducharme. Et celui-ci nous avertit dès le début: "Ce qui est 
souligné se prononce à la française, avec pompe. Ce qui n'est pas souligné se prononce à la québécoise (moi : moin; il: $i$; envoie: enwoueil; je te: j'te; c'est: c'é...)". Ainsi donc, même si le texte, la plupart du temps, est écrit en un français "correct", le passage doit être prononcé à la québécoise s'il n'est pas souligné; et l'association «à la française/avec pompe» nous en dit long sur les intentions parodiques de Ducharme, d'autant plus que la première phrase de l'œuvre: «Elvire, mon chou, m'as-tu fait un rapport bien sincère?" est soulignée - le vers de Corneille, brisé par ce "mon chou», risque fort de sombrer dans le ridicule. Comment le populaire "mon chou" pourrait-il en effet s'accommoder d'une prononciation pompeuse (pompier)?

Dès les premiers instants de la pièce donc, le langage employé par Ducharme nous indique un décalage: entre les vers cornéliens prononcés à la française et la réalité des êtres que Ducharme veut nous présenter, il n'y a pas de commune mesure. Pas plus qu'entre les costumes d'époque et les meubles 1967. Le langage cornélien, ici, devient une illusion grotesque. Et c'est d'ailleurs ce qui frappe d'abord, dès ce premier rideau: le monde dans lequel vivent les personnages est celui de l'illusion que l'on cherche par tous les moyens à entretenir:

Fais-moi-le encore accroire ce que tu viens de me faire accroire. Ah! donne-moi l'illusion d'être là !

Il ne s'agit pas ici de rassurer un cœur inquiet; Chimène, au fond, demande à Elvire de lui raconter des histoires, de lui créer un univers intéressant dans lequel elle pourrait vivre une vie plus exaltante; à la limite, on pourrait dire que la Chimène de Ducharme demande à Elvire de la transporter dans l'univers cornélien (ou dans le monde des romans illustrés?). Mais comment cela serait-il possible, puisque tout le dialogue de ce premier rideau repose sur des situations fausses? Ainsi, quand Elvire, rendant compte de l'entretien qu'elle a eu avec le Comte, dit à Chimène: «Je lui ai dit: “Ce qui compte pour Chimène, c'est de faire plaisir à son père ". II a été bien content de ça...", nous sommes placés au cœur d'une situation entièrement fondée sur et par l'illusion. Car Chimène a déjà dit auparavant: “Dis-moi-le encore qu'on va l'avoir notre licence et qu'on va pouvoir faire ce qu'on a à faire sans aller se cacher au motel Sunset", et le Comte lui-même aurait dit à Elvire: "Si c'est le gars de ce gars-là qui prend le plus de place dans le cœur et dans les culottes de ma fille, tant mieux, je suis bien content." Nous pouvons d'ailleurs croire qu'Elvire rapporte fidèlement à Chimène les paroles de son père, puisque celuici dira plus tard à Rodrigue: «Est-ce qu'elle est bonne dans le bed, ma Chimène?" (Rideau IV, scène 2). Donc Chimène appartient déjà à Rodrigue, le Comte le sait et ne s'en formalise pas: comment pourrait-il croire que «ce qui compte pour Chimène c'est de faire plaisir à son père", comment pourrait-il croire que son approbation du choix de sa fille est autre chose qu'une formalité à laquelle il doit se soumettre? Et comment Chimène pourrait-elle se faire croire (et nous faire croire) que l'avis de son père a une importance quelconque? II s'agit, tout au plus, 
de normaliser une situation qui n'a même pas besoin de l'être, sinon pour éviter quelques embêtements (et pour satisfaire au scénario cornélien).

Se donner l'illusion de, tenter de donner l'illusion de: telle est bien l'une des caractéristiques du comportement de Chimène, mais de Chimène seule (ou presque; l'Infante étant, sur ce plan, le «moule» de Chimène), dans l'couvre de Corneille. Tout au long de la pièce, après le meurtre de son père, Chimène demande en effet la condamnation de Rodrigue tout en souhaitant ne pas l'obtenir; son jeu sera cependant mis à jour (Acte V, scène 6) et Chimène réussira mal à échapper à la risée générale. II n'en va pas ainsi dans la pièce de Ducharme: de même qu'ici, et contrairement à ce qui se passe chez Corneille, le ton de comédie se poursuit bien au-delà des premières scènes, de même tous les personnages veulent donner le change, tout en s'acharnant à répéter de très prosaïques et (apparemment) très factuels "Comme ça, c'est ça" ou des "c'est ça qui est ça» (Rideau III). Par exemple, comment croire à la cause de Don Diègue, quand celui-ci demande à son fils: “Va repriser mon honneur! Va raccommoder ma gloire! " D'autant plus que Rodrigue lui demande tout de suite après: "Qui c'est qui t'a donné une tape encore?" (Rideau III, scène 2). "Repriser», "raccommoder», “encore": autant de mots qui détruisent la réalité que le personnage tente d'imposer. Entre la réalité cornélienne, à laquelle l'auteur l'oblige à être fidèle, et la sienne, l'écart est tellement grand que le langage dénonce cet écart plutôt qu'il ne traduit la situation en laquelle les personnages tentent de croire qu'ils vivent. A la limite, cela donne le "Qu'est-ce qui te prend de changer de ton comme ça tout d'un coup ? (Rideau VII, scène 3) que Chimène lance à Rodrigue lors de leur première rencontre, au moment où celui-ci cesse de réciter des répliques soulignées pour adopter le ton québécois; cela donne encore le "Braille parce que ton père est mort, et je vais brailler parce que Rodrigue m'aime pas" (Rideau IX, scène 2) de l'Infante à Chimène. Pas étonnant que le célèbre récit de la victoire sur les Maures devienne, dans la pièce de Ducharme, un simple échange de textes lus (et prononcés à la française) entre le Roi et Rodrigue, et que les stances de l'Infante deviennent un moment clé de la pièce - la comédienne cesse de jouer son rôle pour nous parler de ses problèmes personnels (Rideau XI, scène 2): à la fin, l'illusion devient trop lourde à porter, l'illusion est tellement transparente qu'elle ne peut plus leurrer personne. Inutile de masquer plus longtemps la réalité; car alors la «simplisterie» naïve deviendrait hypocrisie.

Bien sûr, Ducharme procède ainsi de propos délibéré; il n'est pas dupe de l'illusion, pas plus que du décalage qui la dénonce constamment - tout cela est jeu, qui consiste à faire sciemment pénétrer le spectateur dans un univers qui se dénonce lui-même, où l'illusion de la grandeur ne dévoile que la médiocrité, où la parodie des autres (des personnages de Corneille) devient révélation affligeante de soi-même.

De fait, le héros de Ducharme est précisément celui qui «se met en maudit pour rien" (Rideau III, scène 2), ainsi que le dit Don Diègue. 
L'expression est à retenir, d'autant plus qu'elle n'a aucune connotation péjorative pour celui qui la formule; quand il parle ainsi, Don Diègue fait un compliment à son fils - il ajoute même: "Moi itou j'étais comme ça quand j'étais jeune." On ne saurait mieux dire que Rodrigue est le digne fils de son père et qu'il saura, lui aussi, perpétuer la tradition des grands gestes inutiles. II ne faut pas s'étonner qu'il en soit ainsi; cela découle de la conception de l'homme qui est celle de Don Diègue et qu'il formule ainsi, traduisant à sa façon le célèbre "Rodrigue, as-tu du ccur? ": "Es-tu un homme, un vrai, un gorille? (II bat sa poitrine comme un gorille et crie comme Tarzan.) " Plus loin dans la pièce il y aura un autre cri du même genre, proféré par le Comte, et que Ducharme commentera aussi en parenthèses : «(Grand cri d'adolescent qui a la tremblette.) J'ai pas peur!" (Rideau IV). Le Comte venait tout juste de se faire dire par Don Arias: "Tu parles comme un petit gars de quatorze ans qui vient de lire Les Trois Mousquetaires. »

Contrairement aux héros de Corneille, les héros de Ducharme ne semblent avoir aucune cause à défendre, aucun idéal à proposer ou à protéger - tous les gestes sont ici posés sans qu'on sache trop pourquoi, dans une sorte de perpétuel défi enfantin. Cela n'est jamais si clair que dans les paroles de Rodrigue qui terminent la version ducharmienne des stances:

Si je regarde ça comme il faut, mon affaire est pas si compliquée que ça. Je perds ma blonde "anyway", que je tue le père de ma blonde ou que je le tue pas. Je serais bien fou de me priver de tuer le père de ma blonde. (Rideau III, scène 2)

II s'agit de tuer pour tuer, pour le plaisir de tuer - l'univers de Corneille est devenu celui du western et des films de gansters américains, le page de I'Infante devient le valet noir Blackie, et Paul Newman l'image virile par excellence: "De tous les films de Paul Newman, par exemple, il n'y en a qu'un où on le voit s'excuser" (Rideau VI). Al Capone sera bientôt évoqué, Rodrigue parlera de «la mafia de Gormas", et il menacera son père dans le langage des tueurs à gages: "Cinquante-cinq mille! Pas un peseta de moins! Petit chèque certifié sur le bureau de ma chambre à neuf heures à soir! Ou tu vas être obligé de te passer de mes services" (Rideau VIII, scène 2). Plus tôt dans la pièce, Rodrigue, s'adressant au Comte, avait parlé de lui-même en ces termes:

Je suis jeune, il est vrai; mais ce qui compte ce n'est pas le nombre des années, c'est de ne plus avoir la morve au nez. [...] Souviens-toi que Cassius Clay n'avait que dix-huit ans quand il est monté sur le piédestal qu'occupait Rocky Marciano (Rideau IV, scène 2) ${ }^{\mathbf{y}}$.

Vainqueur de Rodrigue, Don Sanche sera lui aussi comparé à un athlète: "Je pourrais aller jusqu'à dire qu'il marche comme Geoffrion patine quand il vient de scorer" (Rideau XII, scène 2) - cette comparaison n'a cependant rien de flatteur pour Don Sanche -, et la pièce se terminera par une série impressionnante de knock-out enregistrés par Chimène: celle-ci, semble-t-il, a pris la relève de Rodrigue-Cassius Clay. 
Monde du cinéma américain, monde du sport ${ }^{10}$ : monde du spectacle dans le mauvais sens du terme, monde du paraître. Rodrigue meurt de par son goût de se donner en spectacle; et ce goût lui-même ne fait que masquer le vide infini qui l'habite et qu'il cherche à meubler. Ce n'est pas sans raison qu'avant de se comparer à Cassius Clay, Rodrigue avait dit que "ce qui compte, c'est de ne plus avoir la morve au nez"; on n'en finirait plus, dans le Cid maghané, de relever les expressions qui renvoient au monde de l'enfance ou de l'adolescence. Aucun des hommes n'y échappe: «Les vieux, c'est comme les enfants", dit le Comte (Rideau IV, scène 2), et il a bien raison ; car Don Diègue, auparavant, s'était exprimé ainsi :

J'ai plus de mère. C'est maudit; c'est à l'âge que j'ai qu'on a besoin d'être protégé. Une chance que j'ai Rodrigue... Mais c'est pas correct que je me fie sur lui... C'est moi le père... L'enfant, c'est lui... Tout est à l'envers (il pleure). Maman! Maman! (Rideau III)

C'est au pied de la lettre qu'il faut prendre les paroles de Chimène: «L'homme viril et masculin que je cherche, il n'est pas ici» (Rideau X, scène 3): les hommes du Cid maghané sont certes des matamores de taille, mais ce sont surtout des êtres impuissants et sans grandeur. Rodrigue est bien un Québécois et un Nord-Américain: ses exploits consistent à boire le plus de gin possible, à conduire sa nouvelle voiture le plus follement possible, à "faire mâcher de la gomme «balloune» aux éléphants" (Rideau XI). Inutilité, vide et dérision. Au "Cid de poche» succédera “le Cid King's size"; la grandeur de Don Sanche repose sur le fait qu'il a réussi à tuer Rodrigue alors que celui-ci était étendu par terre, saoul. Rodrigue, qui s'était pris pour le Cid (Rideau XI), avait trop misé sur la réputation d'invincibilité de son personnage d'identification et n'avait pas assez songé à se donner une consistance personnelle véritable.

Et puis, les personnages du Cid maghané ne peuvent avoir d'avenir s'ils ne changent pas: le sexe, la «cochonnerie" ainsi que le dit Chimène (Rideau XIII) et l'argent (sur ce plan-là aussi Rodrigue s'avère le digne fils de son père) y occupent trop de place pour que Ducharme puisse réussir à les sauver. Dans ce monde de faux mâles, il est significatif que Chimène, à la fin de la pièce, prenne presque toute la place: débarrassée de la nécessité du paraître et des désirs de Rodrigue, elle sait ramener les mâles à leur réalité de pantins désarticulés, dont le Rói est sans doute le plus parfait prototype. Blackie aussi se fâchera à la fin de la pièce: les dominés en ont assez de devoir correspondre à une image imposée par de si dérisoires dominateurs.

\section{LE CID MAGHANÉ: ESSAI D'INTERPRÉTATION}

Juste avant d'écrire le Cid, Corneille avait écrit une comédie, l'I/lusion comique, dont on a souvent noté la parenté avec le Cid. Doubrovsky en parle ainsi : 
On s'est aperçu qu'à peu de choses près, le langage de Rodrigue est celui de Matamore. S'agirait-il d'une espèce d'humour cornélien, qui saurait au besoin regarder les ressorts de son propre héroïsme d'un œil amusé? [...] C'est exactement l'inverse qui se produit: ce que Corneille expose à la risée, c'est la contrefaçon de l'héroïsme, un héroïsme impur, qui se paye de mots. [...] Si l'on veut, Matamore, c'est le fantôme que Rodrigue incarnera par l'affrontement de la mort. La norme de vérité trouvée, le "Meurs ou tue" de Don Diègue ayant enfin éclaté dans le désert spirituel, en un mot l'être de l'homme étant reconquis sur le paraître, le théâtre se supprime en tant que conscience d'être théâtre, il adhère de nouveau pleinement à lui-même. C'est l'instant où la comédie se consomme et s'accomplit dans la tragédie (CDH, p. 531-532).

Ces lignes ont une très grande importance; d'autant plus que le passage de la comédie à la tragédie, chez Corneille, est aussi lié à la découverte de l'Histoire, à la conscience qu'ont les personnages de s'inscrire dans une continuité historique qui a certes ses lois propres, mais que l'homme a également le devoir de maîtriser. Parodiant le Cid de Corneille, Ducharme recrée Matamore ${ }^{11}$ et non pas Rodrigue, le «héros" de l'illusion et de la mascarade, non pas celui chez qui les gestes ont un sens réel et une portée spécifiquement recherchée. Pour que la tragédie puisse naître, elle doit pouvoir s'inscrire au cœur de l'Histoire; Ducharme tentera cette aventure dans le Marquis qui perdit: notre Histoire même semble si pauvre qu'elle ne réussit pas à donner naissance à une "belle" tragédie.

Là comme à la fin du Cid maghané, nous ne pouvons que «rire jaune", la drôlerie tournant au tragique. Ce n'est pas sans raison que les dernières scènes du Cid maghané sont beaucoup plus près de Beckett et de lonesco que de Corneille: le langage se désagrège et tourne à vide parce qu'il n'essaie plus de donner le change. Rodrigue-Matamore a été détruit par le spectacle, ainsi que le héros de l'lllusion comique. Son paraître a trahi le manque qu'il s'efforçait pourtant de camoufler, qu'il avait pour mission de camoufler (mais de révéler aussi).

Quand Ducharme, infidèle en cela au déroulement du Cid de Corneille, fait mourir Rodrigue dans les circonstances et l'attirail que l'on sait, il nous dit clairement que ce «fendant", préoccupé surtout par l'opinion de ses compagnons de taverne, est davantage un héros à la Paul Newman et à la Cassius Clay qu'un héros selon le Cid: un héros pour la galerie, un héros qui s'efforce de ressembler à de trop multiples modèles, à la seule attitude extérieure et consommable ${ }^{12}$ de trop de modèles. Rodrigue a voulu "faire le frais", selon une expression souvent utilisée dans le Cid maghané: elle dit bien que celui qui se conduit ainsi, hautainement et comme un être supérieur, n'est nullement conforme à l'image qu'il offre.

«Ducharme est un excellent lecteur - et un "nouveau critique» de Corneille", écrivait Laurent Mailhot ${ }^{13}$; ce qui déplaît à Ducharme, ce n'est pas le Cid de Corneille, mais bien le Cid traditionnel présenté dans les écoles, le Cid de l'opposition amour-devoir, le Cid grandiloquent tel qu'incarné il y a une dizaine d'années par un film hollywoodien. Constat de Ducharme: entre le Cid de Corneille et nous, il n'y a pas de commune 
mesure, pour l'instant tout au moins; nous n'avons pas, malgré ce qu'ont pu dire ceux qui enseignaient ici les classiques français, une armature historique qui puisse nous faire correspondre à l'expérience de Rodrigue ${ }^{14}$. II nous reste encore à effectuer le passage de Matamore à Rodrigue.

\section{HAMLET}

Mon oncle qui est mon père et ma mère qui est ma tante ${ }^{15} \ldots$

\section{A. HAMLET DE SHAKESPEARE, SELON JEAN PARIS}

«On ne saurait parler du théâtre élizabéthain sans évoquer ce gigantesque bouleversement d'un monde dont un siècle vient de suffire à ruiner l'ordre" (HPF, p. 50); et c'est d'abord par une longue mise en situation (historique, sociale, religieuse, philosophique, politique) que Paris commence son analyse de Hamlet. Le bel ordre patiemment instauré par le Moyen Âge n'existe plus: la Réforme, la découverte de l'Amérique, de même que les travaux de Copernic (la Terre n'est pas le centre du Cosmos - ce n'est pas autour d'elle que tout gravite), de Montaigne (relativisme moral: dans quelle mesure sommes-nous vraiment responsables de ce qui nous arrive?) et de Machiavel (/e Prince: la théorie du "roi de droit divin" n'est qu'un leurre et une duperie) ont ébranlé, sur tous les plans, les croyances et les institutions médiévales fondées sur le principe «que l'ordre humain reflète l'ordre de l'univers, et l'ordre de l'univers, l'ordre de Dieu 2 (HPF, p. 17).

Les longues hésitations de Hamlet sont la traduction de l'incertitude nouvelle, de ce «doute radical et désespéré» (HPF, p. 39); mais elles sont aussi la manifestation d'une volonté très aiguë de comprendre avant d'agir. “Entre la nostalgie de l'unité perdue et la croissante menace du chaos, [Hamlet] nous apparaît comme un suprême effort de l'homme pour recouvrer sa puissance d'affirmation » (HPF, p. 51-52).

Hamlet est-il bien "le chef-d'œuvre du pessimisme" (HPF, p. 11) qu'on en a fait? Réduite à sa plus simple expression, l'intrigue de la pièce pourrait se résumer ainsi: Hamlet, c'est le drame de trois fils qui doivent venger trois pères. Fortinbras fils doit venger Fortinbras père assassiné par Hamlet père; Hamlet fils doit venger Hamlet père assassiné par son propre frère, Claudius; Laerte doit venger Polonius assassiné par Hamlet fils qui croyait alors tuer Claudius. À la fin de la pièce, c'est Fortinbras qui triomphe; Hamlet mourant, qui a reconnu sa propre cause dans celle de Laerte et vice versa, donne son approbation au jeune seigneur de Norvège: la légalité et la légitimité du pouvoir sont restaurées, puisque c'est le fils du premier roi lésé qui monte sur le trône du Danemark.

Si les fils doivent venger leurs pères, ils doivent aussi apprendre à se gouverner sans les ordres de ceux-ci: "Il faut, pour que le temps 
triomphe du chaos et qu'à son tour la manifestation s'empare du temps, il faut que le Fils triomphe de son Père et lui ravisse la puissance et la vie: il faut que le Fils s'empare de la couronne» (HPF, p. 56). On comprendra que Hamlet ne soit pas toujours très sûr des gestes à poser: il lutte à la fois pour et contre son Père. On comprendra aussi que Fortinbras triomphe: synthèse des qualités de Hamlet (puissance de l'esprit) et de Laerte (capacité d'agir), il a accédé à l'autonomie qui lui permet de reprendre la cause de son père, mais selon ses chemins propres. II s'avère à la hauteur de "cette loi que toute existence répète à son échelle: la loi même de sa naissance et de son dépassement » (HPF, p. 56). En lui, connaissance et liberté ont été réconciliées (HPF, p. 76).

Dans sa structure même d'ailleurs, Hamlet témoigne de cette présence décisive des trois fils, et non du seul Hamlet: la pièce s'ouvre et se termine sur et par eux - voir la scène 2 de l'acte I et la scène finale. Analysé dans cette optique "filiale", Hamlet n'est pas une tragédie pessimiste: "Que masque donc cette triple opposition, d'où la vengeance acquiert la rigueur d'une dialectique? Le mythe shakespearien de l'histoire nous en répond: la volonté de surmonter l'emprise du Père mort afin d'accéder au trône comme à la liberté » (HPF, p. 117-118). D'accéder à la liberté dans la légitimité restaurée.

\section{B. HAMLET, PRINCE DU QUÉBEC: ÉLÉMENTS D'ANALYSE}

Passant de Shakespeare à Gurik, nous passons du Danemark médiéval (ou de l'Angleterre élizabéthaine) au Québec de la fin des années 1960 ${ }^{16}$. Car c'est bien à Montréal, P.Q., à l'occasion de l'Exposition universelle de 1967, que Hamlet père, sous les traits du général Charles de Gaulle, président (et créateur) de la cinquième République française, a décidé de se manifester à son fils québécois du haut du balcon de l'Hôtel de ville de Jean Drapeau. Gurik n'essaie pas de donner le change et ne voile pas ses intentions:

Hamlet c'est le Québec avec toutes ses hésitations, avec sa soif d'action et de liberté, corseté par cent ans d'inaction. Autour de lui se meuvent les masques des personnages qui conduisent sa destinée (H, p. 5).

Dans la version publiée de la pièce, il associe le nom de tel politicien ou de telle institution du Canada ou du Québec au nom du personnage de la pièce de Shakespeare: ainsi, Horatio devient René Lévesque, Laerte devient Pierre Elliott Trudeau, le Roi et la Reine devenant pour leur part l'Anglophonie et l'Église. Sur la scène, pas de doute possible: les personnages de la pièce de Shakespeare portent des masques que le spectateur peut facilement identifier. Seul Hamlet, prince du Québec et le Québec lui-même tout à la fois, joue sans masque. Mais ses vêtements, comme ceux des autres personnages ${ }^{17}$, disent son allégeance: sur fond blanc, sa cape est piquée de fleurs de lys bleues. (Hamlet serait-il un prince québécois de l'ancien régime français?) 
Ce qui frappe aussi, à la lecture du Hamlet de Gurik, ce sont les libertés prises par l'auteur vis-à-vis de la pièce de Shakespeare. Contrairement à Ducharme qui, à quelques exceptions près, respectait le déroulement et l'agencement de l'œuvre de Corneille, Gurik n'hésite pas à supprimer bien des scènes et bien des personnages. Sans doute cette façon de procéder est-elle due à une double çause: l'ampleur même de l'œuvre de Shakespeare interdisait presque à Gurik de procéder de la même façon que Ducharme; de plus, voulant donner à sa pièce une signification avant tout politique ${ }^{18}$, Gurik a délaissé ce qui, dans l'œuvre de Shakespeare, ne lui semblait pas répondre de façon immédiate à ses intentions. Moins fidèle que Ducharme à la construction de la pièce dont il se sert, Gurik par ailleurs ne parodie pas cette dernière; ce qui nous donne des dialogues comme le suivant, à la fois admirablement adapté à la situation québécoise et tout à fait fidèle à l'esprit du Hamlet de Shakespeare:

LA REINE [L'Église] - Cher Hamlet, écarte ces sombres nuages et que ton ceil jette des regards amis sur ton Roi. Ne t'obstine pas à chercher, là où l'herbe prend racine, ton noble père. Résous-toi à cette loi commune que tout ce qui vit, meurt.

hamLet [Québec] — Oui Madame, c'est une loi commune.

LA REINE [L'Église] - Si tu le réalises, pourquoi en sembles-tu si affecté?

HAMLet [Québec] - Sembles, Madame? Mais c'est une réalité et non une apparence. La peine qui creuse mon front n'est pas un déguisement et cette boule dans ma gorge n'est pas la pomme péniblement avalée lors de mon dernier repas. Sembles, Madame, mais cette terre vers laquelle se baissent mes paupières, cette terre où il gît, c'est ma terre et ce n'est plus la sienne ( $H, p .12-13)$.

Gurik a fort bien senti et fort bien rendu, ici, les subtilités de la pensée hamlétienne; surtout, et cet extrait n'est qu'un exemple parmi d'autres, il a choisi avec beaucoup de perspicacité les doubles québécois des personnages de Shakespeare ${ }^{19}$. Ce qui donne aux dialogues de Hamlet, prince du Québec, et au niveau shakespearien et au niveau québécois, une vraisemblance souvent étonnante.

Mais il est bien évident que l'intention première de Gurik est, si l'on peut s'exprimer ainsi, de travailler surtout au niveau québécois de l'œuvre. De traduire le plus fidèlement possible la réalité politique québécoise en se servant de la pièce de Shakespeare, l'utilisation de cette dernière procurant un recul qui donne plus d'impact et de poids à la réalité qu'elle aide à mettre à nu ${ }^{20}$. Aussi, “le rideau s'ouvre sur une scène actuelle" $(H$, p. 5):

... deux fossoyeurs sur le côté de la scène (une colline) jouent aux cartes. Devant eux un trou presque achevé pour recevoir un corps, près d'eux une radio. On devine des monuments funéraires et entre autres la statue de Duplessis $(H$, p. 9).

Il en sera ainsi tout au long de la pièce: même si les masques sont là qui nous disent qu'il faut voir, par exemple, Gérard Pelletier et Jean Marchand en Rosencrantz et Guildenstern, qu'il faut voir l'adéquation 
existant entre le présent et le passé, cela ne semble pas suffire. $A$ intervalles réguliers, les dialogues des fossoyeurs et les nouvelles présentées à la radio nous placeront dans un présent qui n'est que présent, pour nous signifier clairement que nous sommes en pleine actualité, dans le Québec de la fin des années 1960. Ce premier dialogue des fossoyeurs est d'ailleurs une sorte de portrait global de la situation québécoise:

J'suis pas chanceux... revanche des berceaux... Et puis ma femme voudrait pas [prendre la pilule] à cause du curé... Travailler toute la journée parmi les morts, entouré de barrières, c'est pas une vie... J'ai essayé [de faire autre chose] mais j'ai pas d'instruction et puis j'parle pas anglais (H., p. 9-10).

Ainsi résumées, les paroles du 1er Fossoyeur nous présentent tout autant les attitudes avortées du Québec que leurs causes et leurs conséquences séculaires; mais face au 1er Fossoyeur, il y a son compagnon, beaucoup plus hardi et beaucoup plus osé, prêt à se lancer à l'aventure si on veut bien le guider, si on veut bien donner une direction aux forces libératrices qui l'habitent ( $H, p$. 70-72). Dès le début de l'œuvre, nous sommes mis, par le biais des fossoyeurs, en présence des deux attitudes qui opposeront les divers personnages de la pièce: il faut choisir entre l'habitude de la soumission et la volonté de libération.

Les nouvelles présentées à la radio ont encore une autre fonction: ñon seulement replacer les dialogues de Hamlet, prince du Québec dans l'actualité québécoise, mais aussi situer celle-ci par rapport au contexte international. Quand Laerte-Trudeau part pour Ottawa afin de se «parfaire en droit et être digne de [sa] lignée» $(H$, p. 12), la radio présente le message suivant:

... il trouva là un poste qu'il convoitait secrètement grâce à l'appui de Jean Marchand et de l'aile québécoise du parti. Il y a cinq ans jour pour jour éclatait la première bombe séparatiste dans une boîte à lettres. Ce même jour en 1959 le Congo se rebellait pour accéder à son indépen... $(H$, p. 24-25).

D'une part Laerte-Trudeau, soumis, s'en va à Ottawa étudier chez ses maîtres; d'autre part, les bombes séparatistes témoignent d'une volonté de libération que Gurik situe dans le grand mouvement de décolonisation africaine de la décennie 1960. Dans cette optique, le choix que fait Gurik de l'événement primordial de sa pièce est plus qu'heureux: moment privilégié dans la marche vers un Québec libre, «l'apparition» du général de Gaulle a obligé les Québécois à se dévoiler, à opter pour la soumission ou la liberté, à indiquer s'ils étaient du type $1^{\mathrm{er}}$ ou $2^{\mathrm{e}}$ fossoyeur (!); mais elle a aussi révélé (malgré elle?) le vrai visage des anglophones du pays $(H$, p. 33-34) et placé les revendications québécoises dans un contexte international.

Mais revenons à notre point de départ: Hamlet de Shakespeare, avons-nous dit, est le drame de trois fils qui doivent venger trois pères et tout est mis en branle par l'apparition de Hamlet père à Hamlet fils. Au moment de cette apparition, Hamlet père est habillé du costume qu'il portait quand il avait tué Fortinbras père - et l'on sait comment la pièce 
se termine. Mais voilà: Gurik, suivant en cela les traces de bien des metteurs en scène de Hamlet ${ }^{21}$, a supprimé le personnage de Fortinbras. Que va donc dire Hamlet père à son fils québécois - il va parler de vengeance bien sûr, mais de quoi se sentira-t-il lui-même coupable puisqu'il n'a usurpé le trône de personne? Et comment, dans quel esprit son fils exécutera-t-il la vengeance?

«De nouveau revêtu de [ses] habits de combat» $(H$, p. 26), le SpectreCharles de Gaulle explique d'abord à Hamlet-Québec que «le temps n'est pas aux atermoiements mais à la justice» $(H, p .28)$, et qu'il doit venger un meurtre horrible, "le plus horrible commis depuis les jours d'Abraham, alors que la plaine elle-même a perdu son sang» $\left(H\right.$, p. 29) ${ }^{22}$; suit alors la description du meurtre de Hamlet-de Gaulle par son frère, le RoiAnglophonie. Mais surtout, le Spectre dit ceci:

Je t'ai délaissé alors que tu avais le plus besoin de moi. Les soucis de ma charge ne sont pas une excuse suffisante. J'ai été un mauvais père, tu as grandi orphelin et aujourd'hui encore je me présente à toi le visage rougi par les flammes et la honte [...]. Mais tu es ma chair et mon sang... $(H$, p. 28)

En clair, le Père vient dire à son fils ce que le fils ressent: depuis Crémazie jusqu'à Thériault, Langevin et Victor-Lévy Beaulieu, la littérature québécoise a fait amplement usage de ce que Gérard Bessette appelle "l'orphelinisme "23. Bien évidemment, c'est le délaissé qui se sent orphelin; c'est le Québécois qui, depuis 1760, a graduellement développé ce complexe et lui a donné des proportions nationales. Ici, ce n'est pas le fils qui se sent orphelin, c'est le Père qui s'accuse d'avoir laissé grandir son fils dans cet état: Gurik prête au Père le langage du fils, le Père formulant, en termes de remords personnels, ce que le fils a vécu sur le mode du complexe. On ne saurait trouver plus bel exemple de projection, on ne saurait mieux dire que Gurik a écrit sa pièce dans une optique québécoise (ou, à tout le moins, que le Père a adopté, dans son cuvre, l'optique filiale). La réaction de Hamlet-Québec maintenant:

Je me souviens... [...] Mon oncle a tué mon père et usurpé son trône, sa couche, et foule cette terre où il est un étranger. Oui, toute cette angoisse, ce ressentiment d'oppression, d'esclavage [...], je les portais en moi et il a fallu que mon pauvre père rongé par les ans et les remords vienne m'ouvrir les yeux que je tenais fermés. Mon oncle, retenez bien ceci : "Je me souviens, je le jure" $(H, p .30)$.

L'étranger dont parle Hamlet-Québec, c'est bien celui de Patrice Lacombe (la Terre paternelle) et de Félix-Antoine Savard (Menaud, maître-draveur); et il aura fallu l'intervention du Père pour que Hamlet s'ouvre les yeux. Sa devise devient la devise nationale: "Je me souviens", essentiellement tournée vers le passé ${ }^{24}$. Et le passé, en l'occurrence, nous renvoie à la France d'avant la Conquête.

.II y a eu meurtre et usurpation; il y a aussi eu inceste: la ReineÉglise a épousé le meurtrier de son mari. Si rapidement, d'ailleurs, que Hamlet distingue mal le moment des obsèques de son père de celui du remariage de sa mère $(H, p$. 16). Si Hamlet-Québec, avant l'apparition 
du Spectre pouvait affirmer à sa mère:

Vous avez guidé sagement mes pas alors que mes jambes étaient trop frêles pour me porter. Aujourd'hui, encore, je vous écoute et vous obéis. $(H$, p. 13)

les choses ne seront plus les mêmes après les paroles de son père:

LA REINE [L'Église] - Avez-vous oublié qui je suis? J'ai guidé vos premiers pas.

HAMLET [Québec] - Et vous m'avez brouillé la vue. Vous êtes aussi la Reine, la femme du frère de votre époux et de mon père... vous êtes ma mère $(H, \mathrm{p} .67)$.

Les rapports familiaux, depuis ce remariage, étaient devenus fort embrouillés et fort complexes ${ }^{25}$; maintenant, Hamlet retient seulement que la Reine-Église, si importante dans son enfance, a trahi son père en épousant le Roi-Anglophonie. Dès lors, l'autre amour de sa vie, OphélieLesage, fille de Polonius-Pearson, sera irrémédiablement terni par la trahison maternelle: Hamlet-Québec ne veut plus tomber sous la coupe des valeurs féminines et maternelles (qui représentent les seulee autorités strictement québécoises dans la pièce). Entre Hamlet-Québec et sa mère, les relations n'ont pas ce caractère intime qu'elles ont dans la pièce de Shakespeare.

Dans tous ses agissements après l'apparition du Spectre, HamletQuébec respecte scrupuleusement les ordres de ce dernier. En tout cas rien ne nous indique qu'il ait des raisons sérieuses, si l'on fait abstraction de l'habitude de la soumission, de vouloir se dérober au devoir que lui a imposé son père. Tout au long de la pièce de Shakespeare, Hamlet, pour ajuster son comportement à la réalité, disposait d'une image de référence: Fortinbras, et d'un ami sûr et stoïque: Horatio. Entre les ordres de son père et ses penchants personnels, il ne savait trop quoi choisir, il hésitait; pour se donner du temps, il simulait la folie. Mais si bien (parce qu'il était si déchiré), qu'on pouvait le croire fou. Dans Hamlet, prince du Québec rien de tel: Hamlet-Québec simule la folie, cela est clair $^{26}$. II attend seulement l'occasion propice pour exécuter les ordres de son père... tout en demeurant fidèle à la pièce de Shakespeare. De même que Laerte-Trudeau pour le Roi-Anglophonie, il est al'instrument " $(H$, p. 69 et 79$)$ de Hamlet-de Gaulle. Affrontant Laerte-Trudeau en un combat singulier, il affrontera un délégué du pouvoir, non le pouvoir réel: il affrontera le fils de Polonius, de Polonius-Pearson qu'il a tué croyant tuer le Roi; et le fils de Polonius, comme son père, obéit aux ordres du Roi-Anglophonie.

- «... que vive un Québec libre» $(H$, p. 30), disait le Spectre-de Gaulle à Hamlet-Québec lors de son apparition; "être ou ne pas être libre" $(H$, p. 51), dira Hamlet-Québec dans son adaptation du célèbre monologue du héros shakespearien. Après s'être placé sous le patronnage des Patriotes de 1837 ( $H$, p. 86), Hamlet-Québec affrontera Laerte-Trudeau en un «combat fraternel» $(H$, p. 91 et 92$)$, en toute «loyale franchise» $(H, p$. 92) ainsi que l'espérait le Roi-Anglophonie qui avait misé sur la naïveté de 
Hamlet $(H$, p. 80$)$ : Hamlet-Québec et Laerte-Trudeau sont bien deux fils incapables de déjouer les ruses du Père. L'arbitre du combat: HoratioLévesque. Et c'est vers lui que Hamlet mourant se tournera:

Qui... qui... nous sortira de la fange des compromis, de l'esclavage, qui brisera les chaînes qu'hypocritement nous avons nous-mêmes forgées. Il faut que ma mort serve aux autres. II faut... que vive... un... Qué... libre $(H$, p. 95).

Fin bien ambiguë, on l'avouera: sur le point de mourir, Hamlet reprend textuellement les paroles de son père - la situation n'a pas changé. II ne peut qu'espérer que sa mort serve d'exemple, il ne peut que souhaiter la venue d'un Sauveur. Et qui est cet Horatio-Lévesque qu'il retient près de lui? Un être très pondéré, hésitant même $(H, p .26-27$ et 91$)$ qui a déjà "cru pouvoir collaborer à la bonne marche de l'État» $(H, p .16)$, mais qui est revenu de ses illusions. Il a donc su, lui, déjouer les ruses du Pouvoir: c'est la plus sûre garantie qu'il puisse offrir.

\section{HAMLET, PRINCE DU QUÉBEC: ESSAI D'INTERPRÉTATION}

Non plus trois, mais deux: Hamlet, prince du Québec est le drame de deux fils qui doivent venger deux pères - nous sommes jeunes, notre passé ne remonte pas aussi loin dans l'Histoire que celui des Danois ou des Anglais. Il n'y a jamais eu, ici, qu'une seule usurpation, celle de 1760. Remonter plus haut, ce serait nous confondre avec l'Europe de nos origines ${ }^{27}$.

Mais c'est bien jusque-là, ou tout près, que Gurik nous fait remonter: le père de Hamlet-Québec est bien le père de nos origines nationales, le Président, sinon le Roi de la France. A partir du moment où ce père «qui a trop tardé» $(H$, p. 17-18) se manifeste finalement, Hamlet-Québec, semble-t-il, possède la clef des énigmes: le Père a parlé, Hamlet-Québec écoutera sans beaucoup d'hésitations si on le compare à son modèle danois. Il est significatif, en tout cas, que Gurik ne prête pas à HamletQuébec la volonté de tout connaître, la jeunesse studieuse tout autant que les interrogations métaphysiques, qui font que Hamlet le Danois a de sérieuses réserves vis-à-vis de la mission que lui a confiée son père, tout en sachant qu'il devra l'accomplir. Beaucoup plus que son modèle danois, Hamlet-Québec se comporte comme un fils heureux que le Père se soit enfin manifesté et ait indiqué la voie à suivre. Le passé, finalement, a des chances de redevenir le présent.

Surtout, Fortinbras est absent de la pièce de Gurik; Fortinbras par qui se manifeste «la volonté de surmonter l'emprise du Père mort afin d'accéder au trône comme à la liberté» (HPF, p. 117-118). Reste donc Horatio-Lévesque, dont nous avons parlé tout à l'heure. Si HoratioLévesque devait accéder au trône, nous aurions alors la situation suivante: le Roi ne serait pas de sang royal, la lignée serait rompue. Cette perspective ne peut être qu'attrayante, dans la mesure où elle manifesterait enfin l'accès à l'autonomie. D'autant plus que Horatio-Lévesque a déjà 
déjoué le Pouvoir et qu'il n'a pas manifesté un enthousiasme inconsidéré suite à l'apparition du Spectre de Gaulle. Mais rien ne nous assure, contrairement à ce qui se passe pour Fortinbras, que Horatio-Lévesque règnera désormais sur le Québec.

Dans l'ensemble de Hamlet, prince du Québec, Gurik transforme peu la réalité politique québécoise et canadienne, sinon pour un seul fait: au moment de la visite du général de Gaulle, c'est Daniel Johnson et non Jean Lesage qui était premier ministre du Québec. Dans l'œuvre de Gurik $(H, p .55)$, Johnson ne remplace Lesage qu'au cours des événements de 1967. Bien sûr, Gurik a le droit d'agir à sa guise avec les événements; mais justement, il ne le fait pas, sauf pour ce cas-là. Au fond, Gurik veut signifier une chose très simple: le père usurpateur, au Québec, a de multiples visages, de multiples ramifications. Et c'est le Parti libéral qui les regroupe tous et toutes, pour mieux servir l'Anglophonie. Ceux qui servent le Parti libéral ne sont aussi que des fils mal avertis des ruses du Père: des naîfs du type Laerte-Trudeau, des soumis intéressés du type Rosencrantz-Pelletier ou Guildenstern-Marchand ${ }^{28}$.

Plus que le drame de deux fils qui doivent venger deux pères, Hamlet, prince du Québec, à cause des doubles québécois donnés aux personnages de Shakespeare, se présente d'abord à nous comme une grande querelle de famille. II n'y a qu'une famille mais deux pères dont les valeurs sont opposées; le travail des fils ne doit plus être de s'opposer, mais de cesser précisément d'être des fils. De créer Fortinbras ${ }^{29}$, en se débarrassant d'abord du faux père en écoutant le vrai, quitte à se débarrasser aussi du vrai par la suite.

\section{CONCLUSION}

Car si les rois jouent, les comédiens règnent (H, p. 56).

... Le théâtre est le piège Où je prendrai la conscience du roi ${ }^{30}$.

En 1968, nous l'avons dit, deux auteurs québécois choisissent, pour nous parler de nous, de nous présenter un Cid et un Hamlet de leur manière. Cela, à un moment particulièrement important de notre histoire collective, à un moment où, alternativement, notre identité, graduellement redécouverte et dévoilée depuis quelques années, nous est offerte et refusée. Revenons à Doubrovsky:

La structure particulière de "play within a play", de pièce à l'intérieur d'une pièce, que l'on trouve chez Shakespeare, dans l'épisode de la "souricière" hamletienne $[. .$.$] et dont on a toujours souligné la pré-$ sence dans l'lllusion comique, caractérise justement les moments de crise où l'être et le paraître humains se confondent, et où le théâtral devient inextricablement le réel. Pour le pessimisme moderne, l'être n'est pas récupérable sur le paraître; pour Shakespeare et Corneille, la réalité finit par jaillir de la théâtralité. Le vrai Claudius 
paraît, pris dans la souricière; chez Corneille, le Magicien de l'Illusion a seul, en définitive, la clé du réel. Tandis que les aventures de Clindor [et de Matamore] ne nous offraient qu'un simulacre, les exploits de Rodrigue nous transportent au lieu même de la réalité héroïque. ( $C D H$, p. 532)

Par bien des côtés, le Cid maghané et Hamlet, prince du Québec sont précisément des uplay(s) within a play"; de même que Hamlet veut se servir du théâtre comme d'un piège pour prendre la conscience de Claudius, de même Ducharme et Gurik se servent du théâtre pour révéler notre identité; plus précisément, ils se servent d'œuvres théâtrales connues à l'intérieur desquelles ils reprennent et représentent notre aventure ${ }^{31}$ : de même que la souricière joue ce qui fait le sujet de Hamlet, de même Hamlet, prince du Québec, à sa façon, joue ce qui fait le sujet de la pièce de Shakespeare, et à l'intérieur même du cadre fourni par cette dernière. "Le théâtral [est devenu] inextricablement le réel»: «les comédiens règnent» parce que, maîtres de l'illusion, ils sont devenus aussi les maîtres-révélateurs de la réalité. Quant à nous, spectateurs du Hamlet de Gurik ou du Cid de Ducharme, nous devenons, tel le Roi de Hamlet et tels nos propres rois-politiciens, les spectateurs de notre propre jeu - il nous reste à accentuer ce jeu jusqu'à la folie ou la catastrophe ${ }^{32}$, ou à transformer la réalité-jeu en un monde habitable qui ne soit plus aliénant. Où l'être se soit conquis aux dépens du paraître.

“La structure [...] de «play within a play " [...] caractérise justement les moments de crise où l'être et le paraître humains se confondent..." : la décennie 1960, au Québec, ne renvoie-t-elle pas très précisément à ce type de crise? Ne témoigne-t-elle pas d'une volonté de faire le départ entre le paraître et l'être, entre ce qu'on nous disait que nous étions et ce que nous étions (et sommes) en réalité ? Le Cid maghané et Hamlet, prince du Québec nous disent quoi, sinon la nécessité de secouer la torpeur et de mettre fin aux gestes vides, sinon la nécessité de retrouver une autonomie qui ne soit pas illusoire? En ce sens, l'entreprise de Ducharme et Gurik n'est pas bien différente de celle de Jacques Ferron dans les Grands Soleils: dans les trois cas, le théâtre devient «un appareil de sédition ${ }^{33}$ ", Ducharme et Gurik se servant de l'histoire littéraire, si l'on peut parler ainsi, comme Ferron se sert de notre histoire - pour mieux montrer nos visages multiples et nos attitudes souvent contradictoires, et pour tenter de distinguer le vrai du faux dans tout cela.

Ce recours au passé, chez Ducharme et Gurik, peut avoir aussi un autre sens: montrer dans quelle mesure notre histoire peut ressembler à celle des autres, dans quelle mesure elle s'en distingue. Si, par Gurik, nous pouvons constater que la situation politique québécoise ressemble étrangement à celle que décrit le Hamlet de Shakespeare, le Cid maghané nous montre bien qu'à trop vouloir copier le Cid de Corneille nous finissons par devenir pur paraître ${ }^{34}$ : notre réalité est autre, différente. Semblables aux autres, différents d'eux: la présence des autres permet la comparaison. Et celle-ci, dans la mesure où elle sous-entend que nous sommes comparables, accrédite notre histoire et accentue notre identité. 
Dans un même mouvement cependant, et bien que les auteurs, chacun à sa façon, aient su prendre leurs distances vis-à-vis de leur modèle, elles nous disent aussi que le Rodrigue québécois n'est toujours que Matamore et que Hamlet, prince du Québec réussit mal à créer son Fortinbras: il faut cesser de se gargariser de leitmotive du type «Qu'ils viennent les maudits si c'est pas des peureux", comme il faut cesser d'attendre les ordres du père; se dire qu'on ne peut pas être toujours des fils, ainsi que l'affirment les personnages de l'Arme au poing ou larme à l'œil de Dominique de Pasquale ${ }^{35}$.

Renald Bérubé

Université du Québec à Rimouski

1. Jan Kott, Shakespeare notre contemporain, Verviers, Marabout Université, 1965 , p. $78-79$. II semble bien que le Hamlet qui commence cette citation devrait plutôt être un Hamlet; d'autant plus que la phrase qui continue le texte que nous citons commence ainsi: "C'est la plus étrange des pièces que..."

2. Sur la question de l'imitation pratiquée par les auteurs d'ici au xixe siècle, voir l'article de Noël Audet, "la Terre étranqère aporopriée". dans Voix et images du pays II, Montréal, Editions Sainte-Marie, 1969, p. 31-42; et celui de Maximilien Laroche, “Notes sur le style de trois poètes: Roland Giguère, Gatien Lapointe et Paul Chamberland ", ibid., p. 91-106. Laroche écrit ceci: «En imitant, l'on propose de soi une image fausse parce que sans rapport avec le réel, vraie pourtant puisque correspondant à ses désirs et à ses rêves les plus impérieux. L'on escamote la réponse, on devance même la question. L'affirmation sert à cacher l'intırrogation. Le dialogue est truqué. On le dissimule sous des réponses anticipées, plus souhaits que constatations." (p. 94).

3. "La source [de Hamlet] est probablement une vieille légende islandaise retransmise par Belleforest dans ses Histoires tragiques où Šhakespeare l'a sans doute trouvée. Ou peut-être ne faisait-il que réadapter une pièce de Kyd, la Vengeance d'Hamlet, jouée sans doute en 1589 et aujourd'hui perdue. Quoi qu'il en soit, ces sources lui ont fourni la trame de l'intrigue principale qui n'est autre que la conventionnelle vengeance d'un pere (revenge for a father), comme la Vengeance d'Antonio de Marston et bien d'autres pièces contemporaines; car, replacé dans le contexte élizabéthain, Hamlet n'est qu'un exemple entre tant d'autres de ce genre si célèbre à l'époque: la tragédie de la vengeance." (José Axelrad et Michèle Willems, Shakespeare et le théátre élizabéthain, Paris, PUF, "Que sais-je? $\#, n^{\circ} 1096,1964, p .100$.) Voir aussi Jan Kott, op. cit., p. 80.

"Cornei'le cite ses sources dans l'Avertissement: un drame de l'écrivain Guilhem de Castro, joué à Madrid en 1618, las Mocedades del Cid, ala jeunesse du Cid". [...] Ses exploits [de Ruy Diaz de Bivar, modèle du Cid] ont été chantés dans une chanson de geste dès le milieu du xlle siècle, puís au cours du XIIIe, en particulier dans la Chanson de Rodrigue, enfin dans les Romances des XVe et XVle siècles. C'est dans ce Romancero del Cid que Guilhem de Castro puisa l'essentiel de son inspiration. ' (Georges Griffe, dans l'édition du Cid des Petits Classiques Bordas, Paris, 1964, p. 23.)

A noter que le Hamlet de Gurik n'est pas le premier Hamlet québécois; en novembre 1956, Jacques Languirand a fait jouer à la télévision de RadioCanada, un Hamlet... d'après Thomas Kyd!

4. 1968 vit aussi la création du Chemin du Roy de Loranger-Levac, des BellesScours de Michel Tremblay, et les représentations montréalaises des Grands Soleils de Jacques Ferron. 
5. Serge Doubrovskȳ, Corneille et la dialectique du héros, Paris, Gallimard, "Bibliothèque des idées", 1963, 588 p. Chapitre sur le Cid: p. 87-132. Dorénavant, ce livre sera désigné par le sigle $C D H$, et nous donnerons la référence immédiatement après la citation. Pourquoi avoir adopté cette analyse du Cid plutôt qu'une autre? Parce qu'elle semble, dans l'état actuel des recherches, rendre compte de l'œuvre de Corneille mieux que toutes les autres études; elle “lit "l'œuvre de Corneille dans à peu près toutes ses dimensions.

6. Jean Paris, Shakespeare par lui-même, Paris, Seuil, "Ecrivains de toujours", 1961, 192 p.; mais surtout. Hamlet ou les Dersonnages du fils. Paris. Seuil. "Pierres vives", 1953, $188 \mathrm{p}$. Dorénavant, ce livre sera désigné par le sigle HPF, et nous donnerons la référence immédiatement après la citation. Les remarques faites au sujet du Corneille de Doubrovsky s'appliquent au Hamlet de Paris (mais peut-être sont-elles plus subjectives dans ce dernier cas; voir à ce sujet les remarques de Kott, op. cit., p. 85-86).

7. Réjean Ducharme, I'Hiver de force, Paris, Gallimard, 1973, p. 169.

8. Le texte que nous utilisons est celui qui a été joué lors de la création de la pièce. II constitue la troisième version de la pièce. Ce renseignement et le texte nous ont été fournis par $M$. Yvan Canuel, premier metteur en scène du Cid maghané. Puisqu'il ne serait pas très commode d'indiquer la page d'où est extraite telle ou telle citation du Cid maghané, nous donnerons plutôt le rideau et la scène s'il y a lieu.

9. Rodrigue embellit la réalité à son avantage; Cassius Clay n'avait pas dix-huit mais plutôt vingt-deux ans quand il est devenu champion mondial des boxeurs poids lourds. Comme quoi, même en ce qui concerne les événements contemporains, Rodrigue a tendance à s'illusionner pour mieux se donner contenance. Mais peut-être ne faut-il pas oublier que cette phrase est soulignée...

10. Ces deux mondes sont aussi très présents dans l'Hiver de force. Les amis de Nicole et André Ferron appartiennent essentiellement au monde du spectacle que par ailleurs les héros condamnent; le livre contient aussi des allusions aux frères Mahovlich et à la défaite des Canadiens lors des éliminatoires de la coupe Stanley au printemps 1972. De plus, les jeunes Ferron sont des maniaques des films présentés à la télévision, (même si ces films sont le plus souvent très mauvais, à leur grand désespoir): ils regardent toujours les films de fin de soirée. Le Cid maghané ne se termine pas autrement: par des mesures de l'hymne national, après quoi la neige envahit l'écran...

11. Exemples du langage que peut tenir Matamore:

Je te donne le choix de trois ou quatre morts:

Je vais, d'un coup de poing, te briser comme verre,

Ou t'enfoncer tout vif au centre de la terre.

Ou te fendre en dix parts d'un seul coup de revers,

Ou te jeter si haut au-dessus des éclairs

Que tu sois dévoré des feux élémentaires.

Choisis donc promptement, et pense à tes affaires.

(L'Illusion comique, v. 924-930)

Oui, mais les feux qu'il [son fer: son épée] jette, en sortant de prison

Auraient en un moment embrasé la maison,

Dévoré tout à l'heure ardoises et gouttières,

Faîtes, lattes, chevrons, montants, courbes, filières,

Entretoises, sommiers, colonnes, soliveaux,

Parnes, soles, appuis, jambages, travetaux,

Portes, grilles, verrous, serrures, tuiles, pierre,

Plomb, fer, plắtre, ciment, peinture, marbre, verre,

Caves, puits, cours, perrons, salles, chambres, greniers,

Offices, cabinets, terrasses, escaliers.

Juge un peu quel désordre aux yeux de ma charmeuse;

Ces feux étoufferaient son ardeur amoureuse.

(L'Illusion comique, v. 747-758)

12. "ll [Rodrigue] se soûle "à mort", littéralement, et se précipite, skis aux pieds, gants de boxe aux poings, devant l'épée (ou le revolver) de Don Sanche. Son cadavre "beurré de catsup" montre ce qu'était ce jeune homme pour la société: un objet de consommation." (Laurent Mailhot, "le Théâtre "maghané" de Réjean Ducharme", dans Jean-Cléo Godin et Laurent Mailhot, le Théátre québécois, Montréal, Hurtubise H M H, 1970, p. 212.) Objet de 
consommation lui-même modelé sur d'autres objets de consommation (Paul Newman, par exemple): un cycle aussi infernal ne peut mener qu'à un comportement auto-destructeur. Sur ce plan, le Rodrigue de Ducharme est l'exact opposé de celui de Corneille.

13. Ibid., p. 213.

14. Nous rejoignons ainsi ce que disait Michel Van Schendel au sujet de l'Avalée des avalés: "Un critique de mes amis me faisait remarquer que l'Avalée des avalés esquivait soigneusement le sens du tragique. Je ne suis pas loin de penser aujourd'hui qu'il avait raison. La tragédie ne suppose pas seulement une certaine habitude des forces hostiles que l'on affronte et le sentiment de leur irréductibilité. Elle suppose, en plus d'une longue accoutumance historique, d'anciennes traditions, une reconnaissance précise qui se dresse en face de ces forces. Rien de tel chez Réjean Ducharme et cela n'est sans doute pas un hasard. II ne lui appartient pas de créer seul les bases d'une connaissance qui n'apparaît pas encore, ici, comme possible." ("Ducharme I'inquiétant ", dans Littérature canadienne-française, Montréal, PUM, “Conférences J. A. De Sève 1-10", 1969, p. 233.)

Et c'est Ducharme lui-même qui écrit dans le Nez qui voque: "Qu'avaient-ils tous à monter sur leurs cothurnes? Rien ne m'agace plus que le tragique. Je ne trouve rien de plus faux. de plus ridicule, de plus inutile, de plus médiocre que le tragique." (Cité par Laurent Mailhot, op. cit., p. 219.) Le tragique, ici, risque de devenir complaisance masochiste et fataliste; détruire le tragique par le langage ce n'est pas le faire disparaître, c'est en dévoiler les ressorts pour apprendre à le maîtriser. C'est un peu le cheminement suivi par Chimène et I'Infante dans le Cid maghané: personnages de comédie (qui se jouent la comédie) chez Corneille, elles deviennent révélatrices de réalité chez Ducharme.

15. Robert Gurik, Hamlet, prince du Québec, Montréal, Editions de l'Homme, 1968, p. 45-46. Dorénavant cette ceuvre sera désignée par le sigle $H$, et nous donnerons la référence immédiatement après la citation. En 1966, au Festival d'art dramatique de l'Ouest du Québec, Gurik avait déjà présenté une pièce au titre hamlétien: Api or Not Api.

16. Hamlet, prince du Québec a été créé le 17 janvier 1968; on peut présumer qu'il a été écrit à la fin de 1967, après la visite au Québec du genéral de Gaulle (fin juillet 1967). Voyons les hommes qui étaient en place à cette époque. A Ottawa: Lester B. Pearson est premier ministre, mais annonce, le 14 décembre 1967, sa démission cormme chef du Parti libéral. Le parrain du bill omnibus et ministre de la Justice d'alors, Pierre Elliott Trudeau, affirme qu'il ne sera pas candidat à la succession de Pearson. Des " trois colombes" arrivées à Ottawa le 8 novembre 1965, c'est Jean Marchand, ministre de la Main-d'œuvre et de l'Immigration, qui semble le candidat le plus logique car il faut que Pearson ait un successeur francophone. Pourtant, c'est Pierre Elliott Trudeau qui, le 6 avril 1968, à la suite du congrès du Parti libéral, devient le nouveau chef en même temps que premier ministre du Canada. A Québec: Jean Lesage n'est plus premier ministre depuis le 5 juin 1966, date où l'Union nationale de Daniel Johnson a repris le pouvoir. René Lévesque quitte le Parti libéral le 14 octobre 1967 et fonde le Mouvement SouverainetéAssociation quelque temps après; ce n'est qu'en octobre 1968 que sera fondé le Parti québécois. Gurik serait-il prophète sur les bords? II a, de toute façon, fort bien prévu les destins de Marchand et de Trudeau; il a mẻme presque prévu, pourrait-on dire, l'utilisation de la Loi des mesures de guerre d'octobre $1970(H$, p. 64). Et ses rappels du passé des personnages s'avèrent habituellement fort heureux; ainsi, Polonius-Pearson parlant de son fils LaerteTrudeau: " J'ai craint, durant un temps, que ses relations ne l'entrainent dans de folles équipées où se mêlaient le feu et l'amiante. " $(H$, p. 20$)$

17. Ces capes sont intéressantes à détailler: elles révèlent, si l'on peut parler ainsi, les couleurs des personnages. Rosencrantz-Pelletier et Guildenstern-Marchand portent tous deux une cape réversible: aux couleurs du Canada d'un côté, aux couleurs du Québec de l'autre $(H$, p. 41). Selon qu'ils rencontrent Hamlet ou le Roi-Anglophonie par exemple, ils mettront en évidence l'un ou l'autre côté de leur cape. Double costume, double identité: manque d'identité. Quant à Horatio-Lévesque, sa cape montre le symbole de l'Hydro-Québec qui est l'ancêtre, en quelque sorte, de celui du Parti québécois.

18. Ce qui est son droit le plus strict: "Hamlet contient bien des problèmes: la politique, la violence et la morale, la querelle sur l'unité de signification 
de la théorie et la pratique, sur les fins dernières et le sens de la vie; c'est une tragédie d'amour, une tragédie familiale, nationale, philosophique, eschatologique et métaphysique. Tout ce que vous voudrez! Et c'est en plus une bouleversante étude psychologique. Et une intrigue sanglante, un duel, un grand carnage. On peut choisir. Mais il faut savoir dans quel but et pour quelle raison choisir." (Jan Kott, op. cit., p. 74-75) Jan Kott écrit encore: «Car c'est l'époque qui distribue les rôles. Chaqué époque à son tour. C'est elle qui envoie ses Polonius, ses Fortinbras, ses Hamlet et ses Ophélie sur la scène. [...] Chacun des Hamlet a un livre à la main. Mais quel est le livre lu par le Hamlet d'aujourd'hui? Le Hamlet du spectacle cracovien, en cette fin d'automne 1956, ne lisait que des journaux." (Ibid., p. 80 et 83) Le Hamlet québécois, pour sa part, lit 100 Ans d'injustice. $(H, p .39)$

19. Voici les associations établies par Gurik: Hamlet-Québec; le Roi-l'Anglophonie; la Reine-l'Eglise; Polonius-Pearson; Ophélie-Jean Lesage; Laerte-Trudeau; Horatio-René Lévesque; Guildernstern-Jean Marchand; Rosencrantz-Gérard Pelletier; le Spectre-Charles de Gaulle; l'Officier du RHIN-Pierre Bourgault; la Reine (chez les comédiens)-Yvette Brind'Amour; les Rois (chez les comédiens)-Jean Gascon et Gratien Gélinas. II faut aussi relever la joyeuseté suivante: le rôle du Spectre était tenu par Jean-Pierre Saulnier, fils de Lucien Saulnier qui, à l'époque de la venue de de Gaulle, était le bras droit de Jean Drapeau. On sait la longue réponse que Drapeau fera au discours du général. Comme quoi les relations familiales, au Québec, sont bien aussi complexes que dans la pièce de Gurik. Dernière remarque: tous les rôles étaient tenus par des hommes. Fidélité à la tradition médiévale?

20. Pour cette raison-là même, Gurik se devait d'être le plus fidèle possible à l'œuvre de Shakespeare. La tirant trop de son côté, la forçant trop à dire des vérités québécoises, il aurait détruit le recul et donc nui à l'impact de sa pièce.

21. Par exemple: dans le film dont il était le metteur en scène et la vedette, Laurence Olivier a supprimé le personnage de Fortinbras.

22. Parlant ainsi, le Spectre-de Gaulle nous renvoie aux paroles de la chanson sur laquelle commençait la pièce.

23. Gérard Bessette, Trois romanciers québécois, Montréal, Éditions du Jour, 1973, p. 148.

24. Voir l'article de Maximilien Laroche, «Sentiment de l'espace et image du temps chez quelques écrivains québécois", dans Voix et images du pays VII, Montréal, PUQ, 1973, p. 175-176 plus particulièrement. Ici les paroles dé Hamlet-Québec, sa formulation du «Je me souviens" peut cependant être un peu ambiguë; le Spectre-de Gaulle ayant dit: «... et souviens-toi que vive un Québec libre". Ce qui, on l'avouera, n'est pas très clair: le Québec serait condamné à se souvenir d'un rêve qu'il projette dans l'avenir? Hypothèse: de Gaulle, ici, est un père du même type que celui d'Angéline de Montbrun: dans ce roman, les valeurs du père et celles de la France d'avant 1789 se confondent presque toujours.

25. On pourrait trouver de nombreuses autres phrases du type de celle que nous venons de citer; ainsi: “Celui que j'appelais mon fils a tué votre père." $(H$, p. 78$)$ Les nombreuses difficultés familiales, dans la littérature québécoise, renvoient, comme «l'orphelinisme ", à une situation nationale.

26. Nuançons: peut-être est-ce le fait que la pièce de Gurik n'a pas l'ampleur de celle de Shakespeare, n'est pas aussi élaborée dans tous ses aspects, qui concourt à donner cette impression. A la limite, on pourrait dire que HamletQuébec n'est pas suffisamment développé, comme personnage, pour que nous puissions croire en sa folie.

27. Ou avec les Indiens, me font remarquer, avec raison, Robert Claing et Maximilien Laroche. Les premiers usurpés, en Amérique, furent bien les Indiens. Dans cette optique, le Fortinbras québécois, pour être fidèle à celui de Shakespeare et représenter le pouvoir légitime, ne saurait être qu'indien?

28. "Le peuple paie bien mal, monseigneur ", $(H$, p. 44$)$ dit Rosencrantz-Pelletier à Hamlet-Québec.

29. Hubert Aquin, parlant de la situation québécoise, a souvent évoqué le personnage de Hamlet. Que ce soit dans “Profession: écrivain" (Parti pris, $n^{\circ} 4$, janvier 1964, p. 23-31) ou dans sa "Présentation" de la réédition du livre de Maximilien Globensky, la Rébellion de 1837 a Saint-Eustache (Montréal, Editions du Jour, «Bibliothèque québécoise», 1974, p. 7-9). Dans son dernier roman, Neige noire (Montréal, La Presse, 1974), il est souvent question de 
la pièce de Shakespeare; et Aquin accorde une place de choix à Fortinbras. Serait-ce que l'auteur privilégie le «Je dois maintenant à la fois être et ne pas être" de Kierkegaard, qui sert d'épigraphe au roman, aux dépens du « Etre ou ne pas être "de Hamlet?

30. William Shakespeare, Hamlet (traduction de Yves Bonnefoy), Paris, Le Livre de poche, nos 1265-1266, 1964, p. 76. Dans le même volume: Othello et Macbeth, traductions de Armand Robin et Pierre-Jean Jouve.

31. De même aussi, Loranger-Levac, dans le Chemin du Roy (Montréal, Leméac, "Théâtre canadien ", $n^{\circ} 13,1969$ ), se servent d'une structure connue et populaire, celle d'un match de hockey dans l'ambiance du Forum (de Montréal), pour prendre au piège les différences qui séparent la mentalité québécoise et la mentalité canadienne-anglaise. (Curieusement, cette pièce prend aussi au piège la réalité du hockey lui-même: les Canadiens de Montréal ne sont pas qu'un club de hockey. Mais c'est là une autre histoire...)

32. Est-ce bien un hasard si, au début d'un article sur Menaud, maftre-draveur, André Brochu parle de "l'obsession du to-be-or-not-to-be" de Hamlet? (André Brochu, "Menaud ou l'impossible fête", dans l'Action nationale", novembre 1966, p. 266-291; repris dans André Brochu, I'Instance critique, Montréal, Leméac, "Indépendances ", 1974, p. 247-274). Justement, dans quelle mesure pourrait-on comparer la "folie" de Menaud at celle de HamletQuébec? Dans quelle mesure les deux proviennent-elies d'une admiration trop grande pour le père (les pères, dans Menaud) que l'on s'interdit ou s'empêche dès lors d'égaler?

33. «Le Théâtre, ce n'est jamais gratuit, c'est machiné, prémédité, concerté, c'est un appareil de sédition masqué par les feux des projecteurs et les besoins de l'amusement. Si la représentation d'une pièce a du sens, c'est par la conspiration qu'il y a derrière. Telle est l'idée que je me fais du théâtre..." (Jacques Ferron, les Grands Soleils, dans Theatre I, Montréal, Librairie Déom, 1968, p. 17) Le même Jacques Ferron écrit ailleurs: "La tragédie escamote le plus gros de la difficulté en se défaisant des personnages qui ne lui sont plus utiles. Hamlet s'achève sur une telle hétacombe que, faute de Danois, Shakespeare en est réduit à se servir d'un Polonais de la onzième heure pour tirer le rideau." (Du fond de mon arrière-cuisine, Montréal, Editions du Jour, 1973, p. 13) Le Polonais, c'est un Norvégien de la première heure, Fortinbras.

34. Comme Lisette de Courval, dans les Belles-Sœurs, a la suite de son voyage en France.

35. Dominique de Pasquale, l'Arme au poing ou larme à l'ceil (précédé de Oui, chef), Montréal, Léméac, "Répertoire québécois", $n^{\circ} 36,1973$. 